\title{
Interaction of Histidine-rich Glycoprotein with Fibrinogen and Fibrin
}

\author{
Lawrence L. K. Leung \\ Department of Medicine, Division of Hematology-Oncology, and Specialized Center of Research in Thrombosis, \\ The New York Hospital-Cornell Medical Center, New York, New York 10021
}

\begin{abstract}
Histidine-rich glycoprotein (HRGP) is a human plasma and platelet protein of apparently diverse biological functions. In this study a new interaction for HRGP is described. HRGP specifically interacts with fibrinogen as demonstrated by two independent systems. Using an enzyme-linked immunosorbent assay it was demonstrated that HRGP bound to adsorbed fibrinogen in a concentration-dependent and saturable manner, with an apparent dissociation constant $\left(K_{d}\right)$ of $6.7 \mathrm{nM}$. The binding was specific, reversible, and not mediated by a conformationally altered adsorbed fibrinogen molecule. The interaction was divalent cation-dependent and ionic in nature. The HRGP-fibrinogen interaction was also demonstrated using rocket immunoelectrophoresis. The HRGP-fibrinogen interaction had an effect on the kinetics of conversion of fibrinogen to fibrin as demonstrated by a prolongation of the thrombin time. HRGP also became incorporated into fibrin clots in a concentration-dependent and saturable manner, with an apparent $K_{d}$ of $0.25 \mu \mathrm{M}$. The incorporation of HRGP into fibrin clots occurred in a plasma milieu as demonstrated by the direct incorporation of radiolabeled HRGP into plasma clots and by a significant decrease in serum HRGP levels as compared with plasma levels. HRGP prolonged the lag time phase of fibrin gel formation, and decreased the rate of turbidity rise, as well as the final absorbance of fibrin gels. Since the extent of fibrin polymerization was not influenced by the presence of HRGP, these data suggest that fibrin is distributed over more, but thinner, fibrils in the presence of HRGP. In addition to its potential effect on fibrin polymerization, the HRGP-fibrin interaction may play a role in the cell-cell interactions of platelets and macrophages.
\end{abstract}

\section{Introduction}

Histidine-rich glycoprotein (HRGP) ${ }^{1}$ is an $\alpha_{2}$-glycoprotein in human plasma $(1,2)$. In addition to histidine, this protein is rich in proline and glycine, and these three amino acids are primarily located in one restricted region of the molecule (3). The molecular cloning of the HRGP complimentary DNA has recently been reported and demonstrates significant homology to both antithrombin III and high molecular weight kininogen (4). HRGP appears to have diverse biological functions. It binds

Address all correspondence to Dr. Leung, Stanford University School of Medicine, Division of Hematology, Stanford, CA 94305.

Received for publication 9 August 1985 and in revised form 25 November 1985

1. Abbreviations used in this paper: ELISA, enzyme-linked immunosorbent assay; HRGP, histidine-rich glycoprotein.

J. Clin. Invest.

(c) The American Society for Clinical Investigation, Inc.

0021-9738/86/04/1305/07 \$1.00

Volume 77, April 1986, 1305-1311 several divalent metals with high affinity $(5,6)$. HRGP reduces the binding of plasminogen to fibrin and significantly retards fibrinolysis $(7,8)$. There is increased turnover of HRGP during thrombolytic therapy (9). HRGP binds heparin with high affinity and neutralizes its anticoagulant activity; a significant negative correlation has been found between the anticoagulant activity of heparin and the plasma level of $\operatorname{HRGP}(10,11)$. HRGP is also immunochemically identical with a previously described plasma factor which inhibits autorosette formation between erythrocytes and peripheral T lymphocytes (12). We have previously demonstrated that HRGP is present in human platelets, probably located in the $\alpha$-granules, and is released after thrombin stimulation (13). HRGP forms a specific complex with thrombospondin, a platelet adhesive protein (14). HRGP complexed with thrombospondin is capable of binding heparin and neutralizing the anticoagulant activity of heparin in plasma (14). More recently, it was demonstrated that HRGP can form a trimolecular complex with thrombospondin and plasminogen, and HRGP remains functionally active within this macromolecular complex (15). Thus HRGP may function in metal homeostasis, immunoregulation, and may be important in the modulation of thrombotic and fibrinolytic events. In this study a new interaction for HRGP is reported. HRGP interacts specifically with fibrinogen and is incorporated into fibrin clots. This phenomenon appears to alter the final structure of the fibrin gel, suggesting a further degree of complexity to the biology of this molecule.

\section{Methods}

Materials. p-Nitrophenyl phosphate and type VII calf mucosa alkaline phosphatase were obtained from Sigma Chemical Co., St. Louis, MO. Electrophoresis grade agarose (SeaKem) was purchased from FMC Corp., Marine Colloids Div., Springfield, NJ. A chromogenic thrombin substrate, tosyl-gly-pro-arg-paranitroanalide, was obtained from Vega Biotechnologies, Inc., Tucson, AZ. $\epsilon$-Amino- $n$-caproic acid was purchased from Calbiochem-Behring Corp., La Jolla, CA. Microtitration plates and a Titertek multiscan photometer were purchased from Flow Laboratories, Inc., McLean, VA. All reagents were of analytical grade.

Purified proteins. HRGP was purified from fresh platelet-poor human plasma using methods previously described with some modifications (1, $7,13)$. Since HRGP was susceptible to proteolysis (12), protease inhibitors paranitrophenyl paraguanidinobenzoate chloride (pNGB; ICN Biomedical Inc., Costa Mesa, CA) and phenylmethylsulfonyl fluoride (PMSF, final concentration $10^{-5} \mathrm{M}$; Sigma Chemical Co.) were added in the plasma and included in the entire isolation procedure. $50 \%$ polyethylene glycol, $4,000 \mathrm{~mol} \mathrm{wt}$, was added to a final concentration of $6 \%$ to remove the fibrinogen, and the precipitate removed by centrifugation. The supernatant was processed by adsorption to a CM-cellulose 52 column and elution of $\mathrm{HRGP}$ by $\mathrm{NH}_{4} \mathrm{HCO}_{3}$ was monitored by an ELISA specific for HRGP. The eluate was applied to a DEAE column and the peak fractions re-applied to a CM-cellulose column. Traces of IgG, fibrinogen, and plasminogen were removed by affinity chromatography using insolubilized IgG directed against these contaminants. HRGP, purified by this method, was kindly provided by Dr. Peter Harpel, Cornell University Medical College, New York, NY. When analyzed on sodium dodecyl 
sulfate-polyacrylamide gel electrophoresis, purified HRGP showed a single band with an apparent relative molecular weight $\left(M_{\mathrm{r}}\right)$ of 74,000 when reduced with $2 \%$ dithiothreitol. This was in agreement with recent published data (12). Purified human fibrinogen, free of plasminogen, from IMCO Corp. Ltd. AB, Stockholm, Sweden, was purchased from MICO, USA, New York, NY. Coagulability of this fibrinogen preparation was 99\%. In some studies, chromatographically pure Peak I human fibrinogen was used (kindly provided by Dr. Michael Mosesson, Mt. Sinai Medical Center, Milwaukee, WI) and gave essentially the same results. Purified human thrombin $(7,120 \mathrm{NIH} \mathrm{U/ml)}$ was a kind gift of Dr. John Fenton II, New York State Department of Health, Albany, NY. Purified human albumin was purchased from Calbiochem-Behring Corp.

Antisera. Antisera to purified HRGP were raised in rabbits and absorbed with insolubilized HRGP-depleted plasma as previously described (13). The absorbed HRGP antisera produced a single precipitin arc when diffused against human serum and gave a reaction of immunologic identity between serum and the purified HRGP. The absorbed anti-HRGP IgG did not react by ELISA with purified human fibrinogen, Factor VIII-related antigen, plasminogen, albumin, IgG, fibronectin, or thrombospondin. When used in a Western blot against reduced human plasma, the antibody detected a single major band with $M_{\mathrm{r}}$ of 74,000.

Enzyme-linked immunosorbent (ELISA) binding assays. The assay was performed according to the method of Voller et al. as previously described, with minor modifications (14-16). Microtitration plates were coated with $0.2 \mathrm{ml}$ purified fibrinogen, $1-4 \mu \mathrm{g} / \mathrm{ml}$, in bicarbonate coating buffer ( $15 \mathrm{mM} \mathrm{Na}_{2} \mathrm{CO}_{3}, 35 \mathrm{mM} \mathrm{NaHCO}$, $\mathrm{pH} 9.6,0.02 \% \mathrm{NaN}_{3}$ ) for 3 $h$ at $37^{\circ} \mathrm{C}$. The wells were washed three times with Tris-Tween buffer (10 mM Tris- $\mathrm{HCl}, 0.15 \mathrm{M} \mathrm{NaCl}, 0.05 \%$ Tween $20, \mathrm{pH} 7.4$ ) and purified HRGP (diluted in the same buffer) was added in duplicate for $2 \mathrm{~h}$ at $37^{\circ} \mathrm{C}$. After washing to remove unbound HRGP, alkaline-phosphataseconjugated anti-HRGP IgG prepared as previously described (13) was added at 1:800 dilution for $1 \mathrm{~h}$ at $37^{\circ} \mathrm{C}$. After washing, the substrate $p$ nitrophenyl phosphate was added and color development was followed by repeated readings at $405 \mathrm{~nm}$ in a Titertek multiscan photometer. The formation of the HRGP-fibrinogen complex was expressed as the enzymatic activity of the bound alkaline phosphatase $(\triangle A 405 / \mathrm{min})$.

Double antibody ELISA. A double antibody ELISA was used to measure HRGP levels as previously described (13). Microtitration wells were coated with $0.2 \mathrm{ml}$ anti-HRGP IgG $(5 \mu \mathrm{g} / \mathrm{ml})$ in bicarbonate coating buffer for $3 \mathrm{~h}$ at $37^{\circ} \mathrm{C}$. After washing with Tris-Tween buffer, serial dilutions of HRGP $(10-120 \mathrm{ng} / \mathrm{ml})$ in Tris-Tween buffer containing bovine serum albumin $(1 \mathrm{mg} / \mathrm{ml})$ were added in duplicate for $2 \mathrm{~h}$ at $37^{\circ} \mathrm{C}$. After washing, alkaline-phosphatase-conjugated anti-HRGP IgG was added, followed by the enzyme substrate as described in the above section. The resulting hydrolysis of the enzyme substrate was linear with the concentration of HRGP added to the well up to $120 \mathrm{ng} / \mathrm{ml}$. Control studies using nonimmune IgG-coated wells showed no binding of HRGP. This HRGP standard curve was repeated with each experiment in measuring the plasma and serum levels of HRGP. For the preparation of plasma and serum samples, venous blood was obtained using EDTA (final concentration $5 \mathrm{mM}$ ) as anticoagulant from normal donors who had had no medications during the previous $2 \mathrm{wk} .1 \mathrm{ml}$ of the plasma sample was recalcified with $\mathrm{CaCl}_{2}$ (final concentration $11 \mathrm{mM}$ ) and clotted with thrombin $(2 \mathrm{U} / \mathrm{ml})$ for $1 \mathrm{~h}$ at $37^{\circ} \mathrm{C}$. Serum was separated by centrifugation with a tabletop centrifuge (Beckman Instruments, Inc., Fullerton, CA) for 5 min. Plasma and serum samples were diluted 1:1,000 to 1:2,000 in Tris-Tween buffer for measurement of HRGP levels by the double antibody ELISA. In some experiments, thrombin activity was abolished with phe-pro-arg-chloromethyl ketone (PPACK, final concentration 1 $\mu \mathrm{M}$ ) after clotting for $1 \mathrm{~h}$. HRGP levels measured in these samples were not significantly different from those without the thrombin inhibitor.

Rocket immunoelectrophoresis. Agarose stocks were prepared by melting electrophoresis grade agarose in $0.05 \mathrm{M}$ phosphate buffer, $\mathrm{pH}$ 7.4 , at a concentration of $1 \%$. The melted agarose was cooled to $45^{\circ} \mathrm{C}$ and mixed with the prewarmed anti-HRGP antisera at a final concentration of $0.1 \%$. 3-5 ml of antibody-agarose mixture was layered onto glass microscopic slides. Purified HRGP $(0.7 \mu \mathrm{M})$ was preincubated with purified fibrinogen or albumin at various molar concentrations. 12- $\mu 1$ samples were applied to 4-mm diameter wells and subjected to electrophoresis at $50 \mathrm{~V}$ and $4 \mathrm{~mA}$ for $20 \mathrm{~h}$ at $22^{\circ} \mathrm{C}$. After electrophoresis the agarose gels were extensively washed with saline and water, dried, and stained with $1 \%$ amido black in $40 \%$ ethanol- $10 \%$ acetic acid and destained in $90 \%$ methanol-5\% acetic acid. For studies using radiolabeled fibrinogen, the stained dried gels were subjected to autoradiography.

Radioisotope labeling. Purified proteins were labeled with ${ }^{125} \mathrm{I}$ (Amersham Corp., Arlington Heights, IL) by the modified chloramine $T$ method (17). The radiolabeled protein samples were precentrifuged in a centrifuge (Beckman Instruments, Inc.) for $3 \mathrm{~min}$ to remove potential aggregates before each experiment.

Thrombin time assay. $100 \mu \mathrm{l}$ purified fibrinogen (final concentration $2 \mu \mathrm{M}$ ) was incubated with $100 \mu \mathrm{l}$ purified HRGP or albumin at various molar concentrations in PBS containing $1 \mathrm{mM} \mathrm{CaCl}_{2}$ for $10 \mathrm{~min}$ at $37^{\circ} \mathrm{C}$. $50 \mu \mathrm{l}$ thrombin (final concentration $0.2 \mathrm{U} / \mathrm{ml}$ ) was added and clotting times determined in duplicates or triplicates by a fibrometer. Baseline thrombin time in the absence of HRGP was $\sim 160 \mathrm{~s}$ in this assay.

Fibrin clot studies. $100 \mu \mathrm{l}$ of purified fibrinogen (final concentration $2 \mu \mathrm{M}$ ) was incubated with $100 \mu \mathrm{l}$ of ${ }^{125}$ I-HRGP (specific radioactivity $9,000 \mathrm{cpm} / \mu \mathrm{g}$ ) or ${ }^{125} \mathrm{I}$-albumin (specific radioactivity $11,000 \mathrm{cpm} / \mu \mathrm{g}$ ) at various molar concentrations in $10 \mathrm{mM}$ Tris- $\mathrm{HCl}, 0.15 \mathrm{M} \mathrm{NaCl}, 1 \mathrm{mM}$

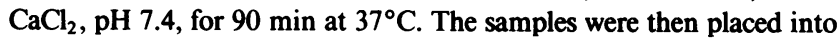
modified 1-ml Eppendorf pipette tips fitted with filter plugs (U-11 plug; Porex Technologies, Fairburn, GA) (18). $50 \mu \mathrm{l}$ thrombin solution (final concentration $0.4 \mathrm{U} / \mathrm{ml}$ ) was added and clotting proceeded at room temperature for $1 \mathrm{~h}$. Unbound proteins were separated by centrifugation for 1 min at 3,000 rpm in a RC-3 centrifuge (Dupont Co., Sorvall Instruments Div., Newtown, CT) and fibrin clots retained on the filters were washed four times with $0.5 \mathrm{ml}$ Tris- $\mathrm{NaCl}$ containing $0.05 \%$ Tween 20 and the amount of labeled proteins incorporated were counted. In some studies, ${ }^{125}$ I-fibrinogen (specific radioactivity $800 \mathrm{cpm} / \mu \mathrm{g}$ ) and unlabeled HRGP were used to determine the extent of conversion of fibrinogen to fibrin.

Fibrin clot absorbance studies. Purified fibrinogen (final concentration $500 \mu \mathrm{g} / \mathrm{ml}$ ) was incubated with purified HRGP or albumin in Tris- $\mathrm{NaCl}$ buffer, pH 7.4, containing $2 \mathrm{mM} \mathrm{CaCl}_{2}$ and trasylol $2 \mathrm{U} / \mathrm{ml}$ for $10 \mathrm{~min}$ at $22^{\circ} \mathrm{C}$. Clotting was initiated by thrombin (final concentration 0.05 $\mathrm{U} / \mathrm{ml}$ ) and the changes in absorbance by the polymerizing fibrin was monitored at $600 \mathrm{~nm}$ in a spectrophotometer.

\section{Results}

Demonstration of HRGP-fibrinogen interaction by ELISA. Using an ELISA binding assay similar to the ones we have previously published $(14,15)$, it was demonstrated that purified HRGP bound to adsorbed fibrinogen in a concentration-dependent and saturable manner (Fig. 1). The binding appeared to be of high affinity, with $50 \%$ binding (apparent $K_{d}$ ) achieved at $\sim 0.5 \mu \mathrm{g} /$ $\mathrm{ml}(6.7 \mathrm{nM})$. Binding of HRGP to fibronectin-coated wells was much less and binding to albumin-coated wells was essentially indistinguishable from that to noncoated blank wells, demonstrating the specificity of this interaction. Using radiolabeled proteins, it was determined that the amounts of fibrinogen, fibronectin, and albumin coated on the wells were comparable (19). Previous studies using a similar ELISA binding assay also showed a minimal interaction of fluid-phase HRGP with adsorbed Factor VIII-related antigen and antithrombin III (14).

To study the time course of HRGP binding to adsorbed fibrinogen, complex formations with different incubation time periods were determined (Fig. 2). A steady state of binding was reached at $1 \mathrm{~h}$ of incubation at $37^{\circ} \mathrm{C}$.

To further demonstrate the specificity of the HRGP-fibrinogen interaction, competitive inhibition studies of HRGP binding to adsorbed fibrinogen by excess fluid-phase fibrinogen were performed (Fig. 3). Fluid-phase fibrinogen, but not fluid-phase albumin, significantly interfered with HRGP binding to adsorbed 


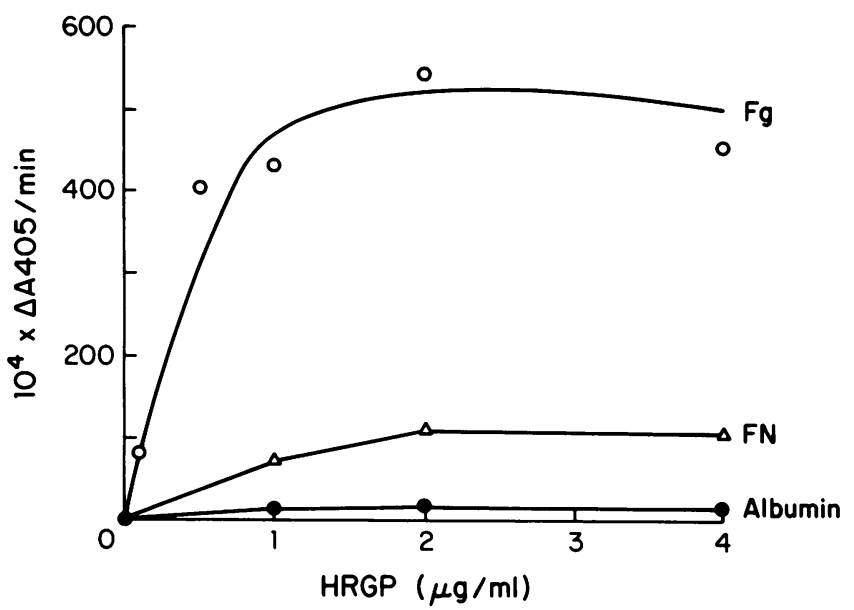

Figure 1. HRGP-fibrinogen interaction. Purified human fibrinogen $(F g)$, fibronectin $(F N)$, and albumin were passively adsorbed to plastic microtiter wells at $4 \mu \mathrm{g} / \mathrm{ml}$ using bicarbonate coating buffer by incubation at $37^{\circ} \mathrm{C} \times 3 \mathrm{~h}$. After washing away unbound proteins, purified HRGP at different concentrations was added in Tris- $\mathrm{NaCl}$ buffer, $\mathrm{pH}$ 7.4 , containing $0.05 \%$ Tween 20 for $2 \mathrm{~h}$ at $37^{\circ} \mathrm{C}$. After washing away unbound HRGP, the amount of HRGP bound to the adsorbed proteins was quantified by the sequential addition of monospecific antiHRGP conjugated with alkaline phosphatase and the enzyme substrate. HRGP bound was expressed as $10^{4} \times \Delta A 405 / \mathrm{min}$.

fibrinogen, with $50 \%$ inhibition occurring at $\sim 6.6 \mu \mathrm{g} / \mathrm{ml}$ fibrinogen (19.4 nM), suggesting that the affinity of HRGP for fluidphase fibrinogen was comparable to that of adsorbed fibrinogen. The data demonstrated that the binding of HRGP to adsorbed fibrinogen was not due to a conformationally altered (denatured) fibrinogen molecule on the plastic surface.

To determine the reversibility of the HRGP-fibrinogen interaction, binding of ${ }^{125} \mathrm{I}$-HRGP to adsorbed fibrinogen was carried out, followed by the addition of excess unlabeled HRGP (Fig. 4). Approximately $80 \%$ of the bound labeled HRGP was displaced into the supernate, demonstrating the reversibility of the interaction.

The binding of HRGP to fibrinogen was divalent cationdependent and was completely inhibited by 5 mM EDTA. There

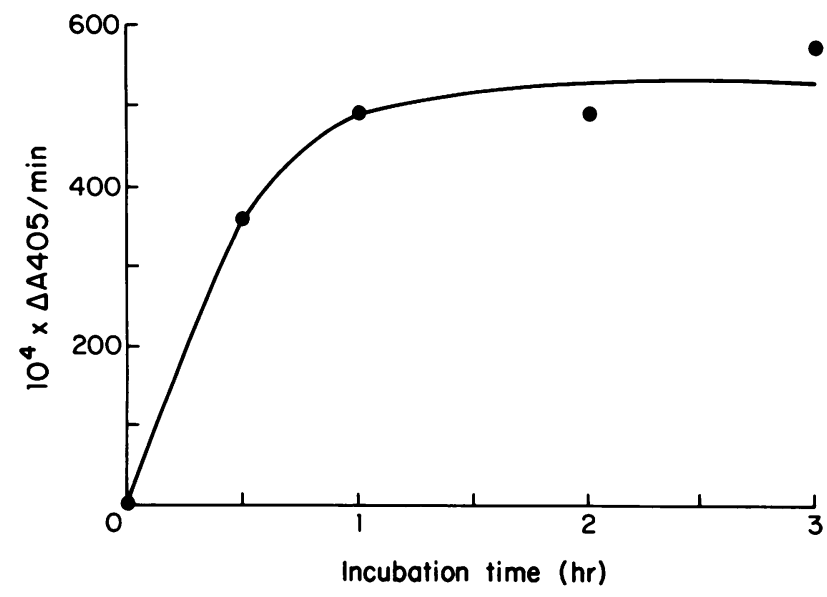

Figure 2. Time course of HRGP binding to adsorbed fibrinogen. Fibrinogen was coated at $1 \mu \mathrm{g} / \mathrm{ml}$ and HRGP at $1 \mu \mathrm{g} / \mathrm{ml}$ was added at $37^{\circ}$ for various periods of incubation time. Binding of HRGP to adsorbed fibrinogen was determined as described in Fig. 1.

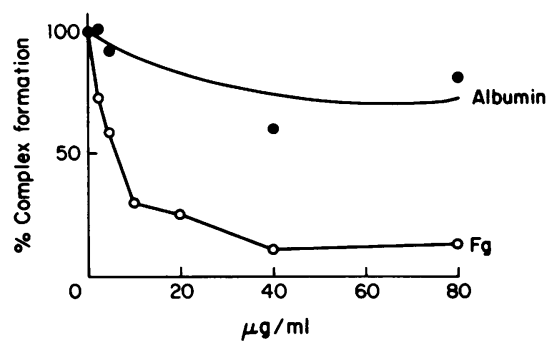

Figure 3. Competitive inhibition of HRGP binding to adsorbed fibrinogen by excess fluid-phase fibrinogen. Purified fibrinogen was coated at $1 \mu \mathrm{g} / \mathrm{ml}$. HRGP $(0.4 \mu \mathrm{g} / \mathrm{ml})$ was added together with purified fibrinogen or albumin at different concentrations for $2 \mathrm{~h}$ at $37^{\circ} \mathrm{C}$. Binding of HRGP to adsorbed fibrinogen was determined as described in Fig. 1. Binding of HRGP in the absence of fluid-phase fibrinogen or albumin was considered $100 \%$.

was no significant difference in the binding of HRGP to EDTAtreated fibrinogen or non-EDTA treated fibrinogen. However EDTA-treated HRGP showed a significant decrease in binding to fibrinogen, suggesting that the calcium dependency was mainly due to a calcium requirement of HRGP in this interaction. The interaction was also affected by the ionic strength of the incubation medium. Binding studies were performed at different ionic strengths, from $0.15 \mathrm{M}$ to $0.5 \mathrm{M} \mathrm{NaCl}$, at increments of $0.05 \mathrm{M}$ $\mathrm{NaCl}$. A 50\% decrease in binding was demonstrated in the presence of $0.25 \mathrm{M} \mathrm{NaCl}$, suggesting that the HRGP-fibrinogen interaction was ionic in nature.

Demonstration of HRGP-fibrinogen interaction by rocket immunoelectrophoresis. To demonstrate the HRGP-fibrinogen interaction by a second system not involving the adsorption of fibrinogen to a plastic surface, rocket immunoelectrophoresis was employed as previously described (15). Purified HRGP was electrophoresed into an agarose gel containing monospecific antiHRGP, giving rise to a well-defined immunoprecipitin arc (Fig. 5). When HRGP was preincubated with purified fibrinogen before immunoelectrophoresis, there was a marked change in the mobility of the HRGP immunoprecipitin arc, demonstrating an

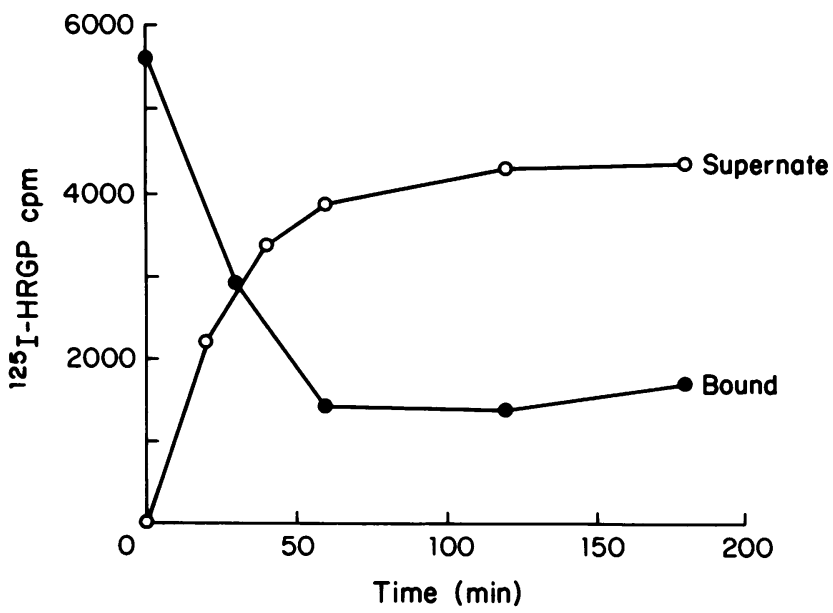

Figure 4. Reversibility of HRGP-fibrinogen interaction. Fibrinogen was coated onto the wells at $2 \mu \mathrm{g} / \mathrm{ml}$. ${ }^{125} \mathrm{I}-\mathrm{HRGP}(0.4 \mu \mathrm{g} / \mathrm{ml})$ was added at $37^{\circ} \mathrm{C}$ for $2 \mathrm{~h}$. After washing away unbound labeled HRGP, excess unlabeled HRGP $(50 \mu \mathrm{g} / \mathrm{ml})$ was added, and radioactivity displaced into the supernatant (as well as that remained bound to the wells) was determined. 


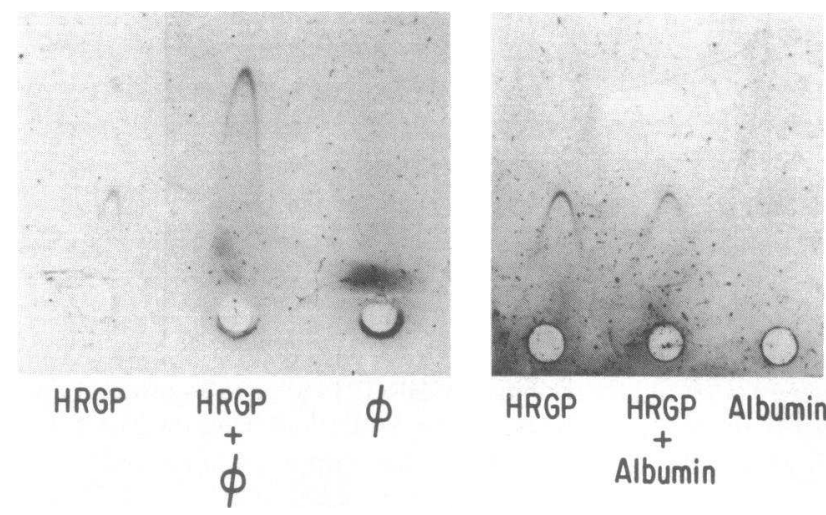

Figure 5. HRGP-fibrinogen immunoelectrophoresis. Purified HRGP (final concentration $0.67 \mu \mathrm{M}$ ) was incubated with purified fibrinogen or albumin (final concentration $5 \mu \mathrm{M}$ ) at $37^{\circ} \mathrm{C}$ for $2 \mathrm{~h}$. The incubation mixtures as well as the purified proteins alone were electrophoresed into agarose containing $0.1 \%$ monospecific anti-HRGP. The agarose gels were extensively washed and stained with amido black.

interaction between HRGP and fibrinogen. When HRGP was preincubated with ${ }^{125}$ I-fibrinogen, incorporation of labeled fibrinogen into the HRGP precipitin arc was demonstrated by autoradiography (data not shown). Purified fibrinogen did not react with anti-HRGP, and control studies using purified albumin showed that it had no effect on the mobility of the HRGP immunoprecipitin arc in this system.

Influence of HRGP on the thrombin time. To explore the functional implications of the HRGP-fibrinogen interaction, the influence of HRGP on the thrombin time was investigated. Purified fibrinogen was preincubated with HRGP at different molar ratios, clotting was initiated with the addition of thrombin, and thrombin times determined (Fig. 6). HRGP caused a significant prolongation of the thrombin time. The prolongation was concentration-dependent. Control studies using purified albumin, at equivalent molar concentrations, had no effect on the thrombin time. HRGP at the concentrations used in this assay system

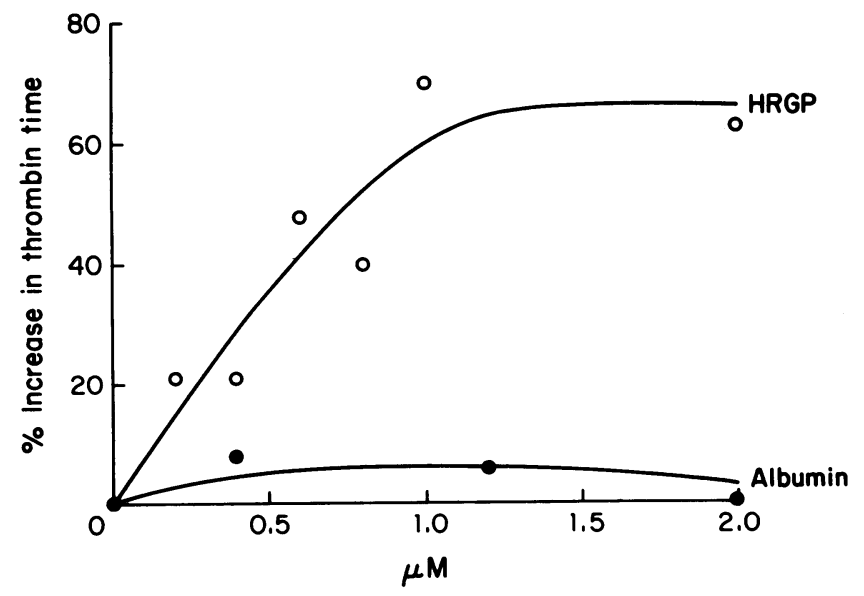

Figure 6. Effect of HRGP on the thrombin time. $2 \mu \mathrm{M}$ of purified fibrinogen was incubated with different concentrations of HRGP or albumin in PBS containing $1 \mathrm{mM} \mathrm{CaCl}$ for $10 \mathrm{~min}$ at $37^{\circ} \mathrm{C}$. Thrombin (final concentration $0.2 \mathrm{U} / \mathrm{ml}$ ) was added and the thrombin times determined using a fibrometer. had no inhibitory effect on thrombin activity as determined by a chromogenic substrate (data not shown).

Incorporation of HRGP into fibrin clots. Studies were performed to determine if HRGP was incorporated into fibrin clots. Purified fibrinogen was incubated with radiolabeled HRGP or albumin at different molar concentrations, fibrin clots were formed by the addition of thrombin, and the amount of radiolabeled proteins incorporated determined (Fig. $7 A$ ). ${ }^{125}$ I-HRGP became incorporated into fibrin clots in a concentration-dependent and saturable manner, with $50 \%$ incorporation achieved at $\sim 0.25 \mu \mathrm{M}$ (apparent $K_{\mathrm{d}}$ ). There was an $80 \%$ decrease in HRGP incorporation into fibrin clots formed in the presence of $5 \mathrm{mM}$ EDTA as compared with $1 \mathrm{mM} \mathrm{CaCl}$, suggesting that it was calcium-dependent. The HRGP incorporation did not represent nonspecific trapping since there was minimal binding of ${ }^{125} \mathrm{I}$-albumin, at equivalent molar concentrations, to fibrin clots. Additional control studies showed that in the absence of fibrin clot formation, the amount of ${ }^{125}$ I-HRGP retained in the filter was $<10 \%$ of that detected in the presence of clot formation, indicating that the HRGP incorporation did not represent non-
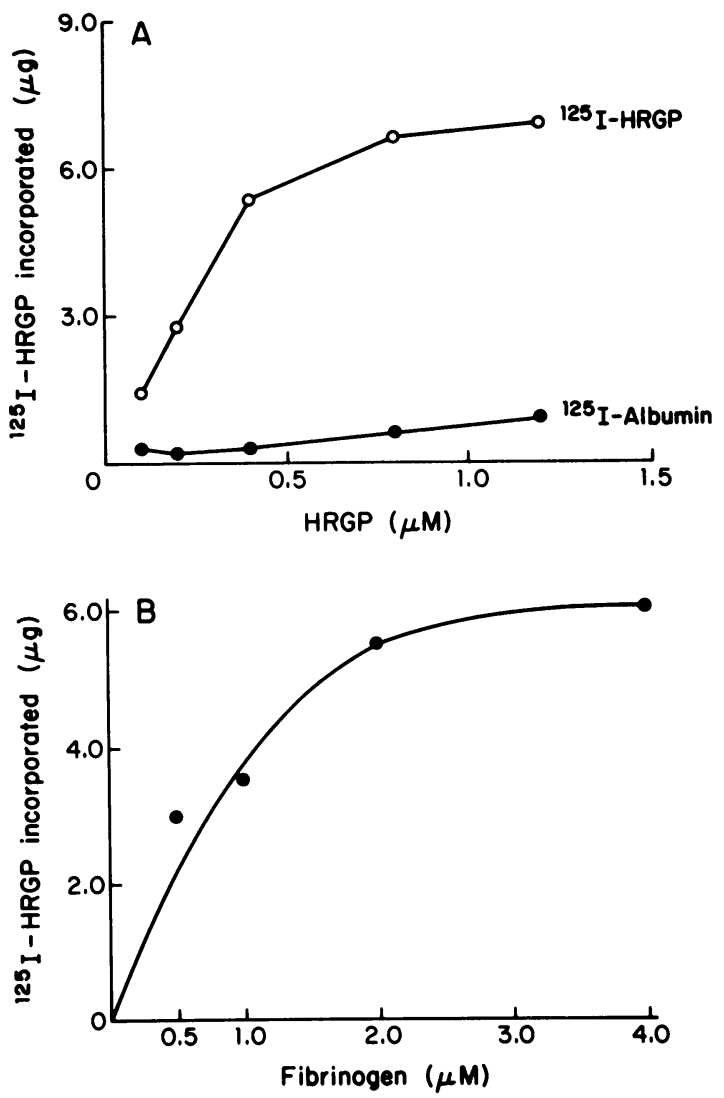

Figure 7. HRGP incorporation into fibrin clots. $(A) 2 \mu \mathrm{M}$ of purified fibrinogen was incubated with various concentrations of ${ }^{125}$ I-HRGP or ${ }^{125} \mathrm{I}$-albumin for $90 \mathrm{~min}$ at $37^{\circ} \mathrm{C}$. The incubation mixtures were placed in modified 1-ml pipette tips fitted with filters (18). Thrombin (final concentration $0.4 \mathrm{U} / \mathrm{ml}$ ) was added and clotting proceeded at room temperature for $1 \mathrm{~h}$. Unbound proteins were separated by centrifugation and the clots extensively washed with $\mathrm{Tris}-\mathrm{NaCl}$ buffer containing $0.05 \%$ Tween 20 and the amount of labeled proteins incorporated in fibrin clots was determined by counting. (B) $0.43 \mu \mathrm{M}$ of ${ }^{125} \mathrm{I}$-HRGP was incubated with various concentrations of purified fibrinogen and the incorporation of labeled HRGP into fibrin clots determined as described in $7 \mathrm{~A}$. 
specific binding of the radiolabeled proteins to the filter. The incorporation of HRGP was also dependent on the concentration of fibrinogen, with $50 \%$ incorporation achieved at $\sim 0.7 \mu \mathrm{M}$ (Fig. 7 B). Using ${ }^{125}$ I-fibrinogen, it was demonstrated that the incorporation of HRGP had no effect on the extent of fibrinogen conversion into fibrin by thrombin. At the optimal concentrations of fibrinogen and HRGP, the mole ratio of fibrin (monomers) and HRGP in the fibrin clot was determined to be $\sim$ 10:1. HRGP-depleted plasma reconstituted with ${ }^{125}$ I-HRGP also showed incorporation of HRGP into the plasma clot, indicating that this interaction occurred in a physiologic milieu. There was no inhibition of HRGP incorporation in the presence of $10 \mathrm{mM} \epsilon=$ amino $=n=$ caproic acid, indicating that the lysine-binding site was not involved in the HRGP-fibrin interaction and that the observed incorporation was not mediated by HRGP binding to a trace amount of contaminating plasminogen in the fibrinogen preparation.

Effect of HRGP incorporation on the structure of the fibrin gel. The effect of HRGP incorporation on the structure of the fibrin gel was studied by opacity. Purified fibrinogen was incubated with different concentrations of HRGP or albumin and clotting initiated by thrombin. The changes in absorbance at $600 \mathrm{~nm}$ by the polymerizing fibrin was monitored (Fig. 8). HRGP caused a slight prolongation of the time required before an increase in absorbance was observed (lag time). It significantly decreased the rate of increase in absorbance as well as the final absorbance of the fibrin gel, which persisted after $24 \mathrm{~h}$ of incubation, suggesting that the HRGP incorporation altered the final structure of the fibrin gel. Purified albumin as a control had no effect.

Plasma and serum levels of HRGP. Since our studies indicated that the incorporation of HRGP into fibrin clots occurred in a plasma milieu, the plasma and serum levels of HRGP were measured to determine if there was a detectable difference. A double antibody ELISA for HRGP was used to measure HRGP levels as previously described (13). The plasma concentration of HRGP was determined to be $126.2 \pm 12.7 \mu \mathrm{g} / \mathrm{ml}(n=10)$, in good agreement with published data $(1,7,13)$. There was an $\sim 20 \%$ decrease in HRGP level in serum, indicating a significant incorporation of HRGP into the plasma clot (Table I).

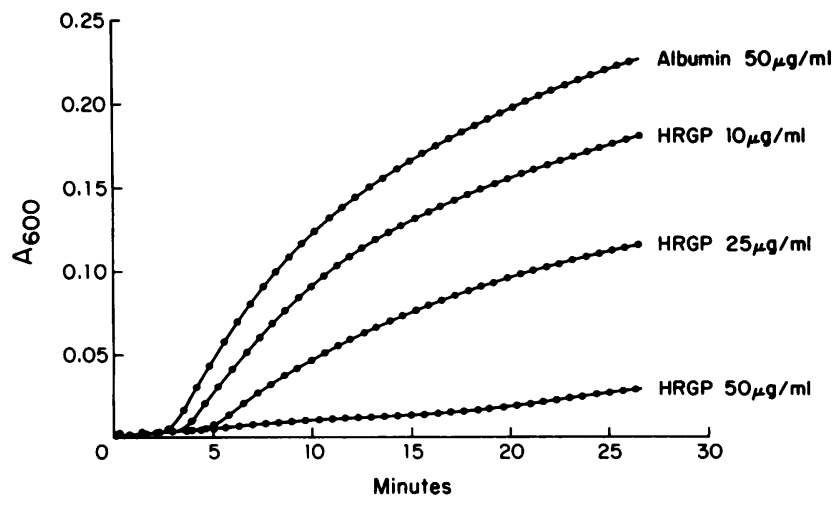

Figure 8. Effect of HRGP on the structure of fibrin gels as monitored by opacity. Purified fibrinogen $(0.5 \mathrm{mg} / \mathrm{ml})$ was incubated with different concentrations of HRGP or albumin for $10 \mathrm{~min}$ at room temp. Clotting was initiated by the addition of thrombin (final concentration $0.05 \mathrm{U} / \mathrm{ml}$ ) and the changes in absorbance at $600 \mathrm{~nm}$ by the polymerizing fibrin followed in a spectrophotometer.
Table I. Plasma and Serum Levels of HRGP

\begin{tabular}{clc}
\hline & \multicolumn{2}{l}{ HRGP level } \\
\cline { 2 - 3 } Donor & Plasma & Serum \\
\hline & $\mu g / m l$ & $\mu g / m l$ \\
1 & 145.5 & 106.5 \\
2 & 121.5 & 110.6 \\
3 & 117.0 & 100.6 \\
4 & 103.5 & 82.8 \\
5 & 121.5 & 96.0 \\
6 & 136.0 & 109.9 \\
7 & 116.8 & 102.4 \\
8 & 140.0 & 101.0 \\
9 & 126.4 & 94.4 \\
10 & 134.0 & 96.0 \\
Mean \pm SD & $126.2 \pm 12.7^{*}$ & $100 \pm 8.3^{*}$ \\
\hline
\end{tabular}

Venous blood was obtained using EDTA (final concentration $5 \mathrm{mM}$ ) as anticoagulant. An aliquot of this was recalcified with $\mathrm{CaCl}_{2}$ (final concentration $11 \mathrm{mM}$ ). The recalcified plasma was clotted by thrombin (final concentration $2 \mathrm{U} / \mathrm{ml}$ ) for $1 \mathrm{~h}$ at $37^{\circ} \mathrm{C}$. Serum was separated by centrifugation in a tabletop microfuge (Beckman Instruments, Inc.) for $5 \mathrm{~min}$. Plasma and serum samples were then diluted 1:1,000 to 1:1,500 and HRGP levels measured by ELISA.

$* P<0.001$.

\section{Discussion}

In this study, a new interaction for HRGP is described. HRGP specifically interacts with fibrinogen as demonstrated by two independent systems, an ELISA binding assay (Figs. 1-4) and rocket immunoelectrophoresis (Fig. 5). HRGP bound to adsorbed fibrinogen with an apparent $K_{d}$ of $6.7 \mathrm{nM}$ (Fig. 1). It is possible that fibrinogen adsorbed on the plastic surface has an altered affinity for HRGP and this may not represent an accurate determination of the dissociation constant. However the competitive inhibition studies by fluid-phase fibrinogen (Fig. 3) suggest that the native fibrinogen molecule has a high affinity for HRGP in the fluid phase. While the stoichiometry of the HRGPfibrinogen interaction remains undefined, the data suggest that a significant proportion of the plasma HRGP molecules may bind to fibrinogen and circulate as HRGP-fibrinogen complexes. The effect of other plasma proteins, such as plasminogen, which may interact with both HRGP and fibrinogen $(7,20)$, remains to be determined. On the other hand, it should be noted that the molar ratio of fibrinogen to HRGP is $\sim 10: 1$ in plasma. It is therefore likely that most of the plasma fibrinogen molecules are not complexed to HRGP.

The HRGP-fibrinogen interaction had an effect on the conversion of fibrinogen to fibrin as demonstrated by a prolongation of the thrombin time (Fig. 6). This was not mediated by an antithrombin effect, as indicated by the lack of inhibition of thrombin activity by HRGP using a chromogenic substrate. In these experiments a maximal prolongation of the thrombin time was achieved at a molar ratio of fibrinogen to HRGP of $\sim 1.6$ : 1. At a plasma molar ratio of fibrinogen to HRGP of 10 : 1 , the prolongation of the thrombin time was $\sim 10 \%$ in the purified system, thus in agreement with the observation that the thrombin times of HRGP-depleted plasma and normal plasma 
were not significantly different $(10,14)$. It suggests that HRGP probably does not have a major effect on the kinetics of fibrin formation in plasma, except perhaps in a microenvironment where there is a high local concentration of HRGP, e.g., in a focal area of active hemostasis and thrombosis where extensive release of platelet HRGP occurs (13).

There was specific binding and incorporation of HRGP into fibrin clots with an apparent $K_{\mathrm{d}}$ of $0.25 \mu \mathrm{M}$ (Fig. $7 \mathrm{~A}$ ). While the assay systems for HRGP binding to fibrinogen and fibrin were different, the data suggest that the affinity of HRGP for fibrin may be lower than that for fibrinogen. Using radiolabeled proteins, it was demonstrated that the HRGP incorporation had no effect on the extent of conversion of fibrinogen to fibrin. At the optimal concentrations of fibrinogen and HRGP, the mole ratio of fibrin (monomers) and HRGP in the fibrin clot was determined to be $\sim 10: 1$ and not stoichiometric. While the reason for this was not determined, it should be noted that fibrin polymerization is a complex process and the properties of fibrin are determined by kinetics and not by equilibria of assembly steps (21). It is possible that the incorporation of HRGP into polymerizing fibrin is controlled kinetically and therefore a nonstoichiometric relationship between fibrin (monomers) and HRGP of 10:1 was obtained. Another possibility is that the HRGP-binding site on the fibrin monomer is located at or near a polymerization domain. Since complementary polymerization domains must have high affinity, fibrin polymerization would lead to the release of HRGP, resulting in a nonstoichiometric mole ratio between HRGP and fibrin monomers in the fibrin clot.

The incorporation of HRGP into fibrin had a significant effect on the final structure of fibrin gels as monitored by opacity (Fig. 8). HRGP prolonged the lag time and decreased the rate of turbidity rise as well as the final absorbance of fibrin gels. The decrease in the final absorbance of fibrin gels suggests that the effect was not mediated by an antithrombin effect, and was consistent with the lack of inhibition of thrombin activity by HRGP using a chromogenic substrate. The final optical density of a fibrin gel is an indication of the average mass/length ratio of fibrin fibers within the gel (22). Since the extent of fibrin polymerization was not influenced by the presence of HRGP, these data suggest that fibrin is distributed over more, but thinner, fibrils in the presence of HRGP. It should be noted that the effect of HRGP on the gel structure was concentration-dependent and was observed when fibrinogen and HRGP were present at molar concentrations of 11:1 (Fig. 8), similar to their molar ratio in plasma, suggesting that this may occur in a plasma milieu. The potential physiological significance of the HRGP-fibrin interaction was also suggested by the direct incorporation of radiolabeled HRGP into plasma clots and the significant decrease in serum HRGP levels as compared with plasma levels (Table I).

A limited number of proteins have been reported to become associated with fibrin or plasma clots, including plasminogen, fibronectin, $\alpha_{2}$-plasmin inhibitor, thrombin, Factor XIII, and more recently, thrombospondin $(20,23-31)$. When fibrinogen is clotted in the presence of plasminogen, the lag time to turbidity rise is decreased, and the rate and extent of turbidity rise are increased, suggesting that plasminogen may bind divalently between two monomer units of forming polymeric fibrin (23). Thrombospondin decreases the lag time to turbidity rise as well as the final opacity, indicating that branching is made more favorable by the presence of thrombospondin (31). HRGP in- teracts with both plasminogen (7) and thrombospondin (14), and HRGP can form a trimolecular complex with plasminogen and thrombospondin (15). It will be of interest to determine the effect of these three proteins together on fibrin polymerization.

The biological significance of the HRGP interaction with fibrinogen and fibrin may not be limited to the fibrin polymerization process. Fibrin is formed on the thrombin-activated platelet surface and we have previously demonstrated the release of platelet HRGP upon thrombin stimulation (13). Recent studies suggest that macrophages may secrete HRGP (32). Fibrinogen-fibrin has been detected on peritoneal macrophages (33), and specific binding of soluble fibrin to macrophages has been reported $(34,35)$. Whether the interaction of HRGP with fibrinogen and fibrin plays a role in the cell-cell interactions of platelets and macrophages remains to be determined.

\section{Acknowledgments}

I am deeply grateful to Dr. Ralph Nachman for his continued valued advice and encouragement. I wish to thank Miss Marilyn Jung for her excellent technical assistance.

Dr. Leung is the recipient of Clinical Investigator Award K08 HL00877 from the National Heart, Lung, and Blood Institute and a Career Scientist Award from the Irma T. Hirschl-Monique Weill-Caulier Trust.

\section{References}

1. Haupt, H., and N. Heimburger. 1972. Humanserumproteine mit hoher affinitat zu carboxymethylcellulose I. Hoppe-Seyler's Z. Physiol. Chem. 353:1125-1132.

2. Heimburger, N., H. Haupt, T. Kranz, and S. Baudner. 1972. Humanserumproteine mit hoher affinitat zu carboxymethyl cellulose II. Hoppe-Seyler's Z. Physiol. Chem. 353:1133-1140.

3. Morgan, W. T. 1985. The histidine-rich glycoprotein of serum has a domain rich in histidine, proline, and glycine that binds heme and metals. Biochemistry. 24:1496-1501.

4. Koide, T., D. Foster, S. Yoshitake, and E. W. Davie. 1985. Tandem repeats in the human histidine-rich glycoprotein as shown by cloning and sequencing of cDNA. Thromb. Haemostasis. 54:168. (Abstr.)

5. Morgan, W. T. 1978. Human serum histidine-rich glycoprotein I. Interactions with heme, metal ions, and organic ligands. Biochim. Biophys. Acta. 353:319-333.

6. Morgan, W. T. 1981. Interactions of histidine-rich glycoprotein of serum with metals. Biochemistry. 20:1054-1061.

7. Lijnen, H. R., M. Hoylaerts, and D. Collen. 1980. Isolation and characterization of a human plasma protein with affinity for the lysine binding sites in plasminogen. J. Biol. Chem. 255:10214-10222.

8. Ichinose, A., J. Mimuro, T. Koide, and N. Aoki. 1984. Histidinerich glycoprotein and $\alpha_{2}$-plasmin inhibitor in inhibition of plasminogen binding to fibrin. Thromb. Res. 33:401-407.

9. Lijnen, H. R., F. De Cock, and D. Collen. 1981. Turnover of human histidine-rich glycoprotein in healthy subjects and during thrombolytic therapy. Thromb. Res. 23:121-131.

10. Lijnen, H. R., M. Hoylaerts, and D. Collen. 1983. Heparin binding properties of human histidine-rich glycoprotein. Mechanism and role in the neutralization of heparin in plasma. J. Biol. Chem. 258:3803-3808.

11. Lijnen, H. R., B. Van Hoef, and D. Collen. 1984. Histidine-rich glycoprotein modulates the anti-coagulant activity of heparin in human plasma. Thromb. Haemostasis. 51:266-268.

12. Lijnen, H. R., D. B. Rylatt, and D. Collen. 1983. Physicochemical, immunochemical and functional comparison of human histidine-rich glycoprotein and autorosette inhibition factor. Biochim. Biophys. Acta. 742:109-115.

13. Leung, L. L. K., P. C. Harpel, R. L. Nachman, and E. M. Rabellino. 1983. Histidine-rich glycoprotein is present in human platelets and is released following thrombin stimulation. Blood. 62:1016-1021. 
14. Leung, L. L. K., R. L. Nachman, and P. C. Harpel. 1984. Complex formation of platelet thrombospondin with histidine-rich glycoprotein. J. Clin. Invest. 73:5-12.

15. Silverstein, R. L., L. L. K. Leung, P. C. Harpel, and R. L. Nachman. 1985. Platelet thrombospondin forms a trimolecular complex with plasminogen and histidine-rich glycoprotein. J. Clin. Invest. 75:20652073.

16. Voller, A., D. Bidwell, and A. Bartlett. 1976. Microplate immunoassays for the immunodiagnosis of virus infections. In Manual of Clinical Immunology. N. R. Rose and H. Freidman, editors. American Association of Microbiology, Washington, DC. 506-512.

17. McConahey, P., and F. Dixon. 1966. A method of trace iodination of proteins for immunologic studies. Int. Arch. Allergy Appl. Immunol. 29:185-189.

18. Harpel, P. C., T.-S. Chang, and E. Verderber. 1985. Tissue plasminogen activator and urokinase mediate the binding of glu-plasminogen to plasma fibrin I. Evidence for new binding sites in plasmin-degraded fibrin I. J. Biol. Chem. 260:4432-4440.

19. Leung, L. L. K., and R. L. Nachman. 1982. Complex formation of platelet thrombospondin with fibrinogen. J. Clin. Invest. 70:542-549.

20. Lucas, M. A., L. J. Fretto, and P. A. McKee. 1983. The binding of human plasminogen to fibrin and fibrinogen. J. Biol. Chem. 258: 4249-4256.

21. Hantgan, R. R., and J. Hermans. 1979. Assembly of fibrin. A light scattering study. J. Biol. Chem. 254:11272-11281.

22. Carr, M. E., Jr., and J. Hermans. 1978. Size and density of fibrin fibers from turbidity. Macromolecules. 11:46-50.

23. Garman, A. J., and R. A. G. Smith. 1982. The binding of plasminogen to fibrin: evidence for plasminogen-bridging. Thromb. Res. 27: 311-320.

24. Hoylaerts, M., D. C. Rijken, H. R. Lijnen, and D. Collen. 1982.
Kinetics of the activation of plasminogen by human tissue plasminogen activator. J. Biol. Chem. 257:2912-2919.

25. Mosher, D. F. 1975. Cross-linking of cold-insoluble globulin by fibrin stabilizing factor. J. Biol. Chem. 250:6614-6621.

26. Niewiarowska, J., and C. S. Cierniewski. 1982. Inhibitory effect of fibronectin on the fibrin formation. Thromb. Res. 27:611-618.

27. Sakata, Y., and N. Aoki. 1980. Cross-linking of $\alpha_{2}$-plasmin inhibitor to fibrin by fibrin-stabilizing factor. J. Clin. Invest. 65:290-297.

28. Sakata, Y., J. Mimuro, and N. Aoki. 1984. Differential binding of plasminogen to cross-linked and noncrosslinked fibrins: its significance in hemostatic defect in factor XIII deficiency. Blood. 63:1393-1401.

29. Kaminski, M., and J. McDonagh. 1983. Studies on the mechanism of thrombin. Interaction with fibrin. J. Biol. Chem. 258:10530-10535.

30. Curtis, C. G., K. L. Brown, R. B. Credo, R. A. Domanik, A. Gray, P. Sternberg, and L. Lorand. 1974. Calcium-dependent unmasking of active center cysteine during activation of fibrin stabilizing factor. Biochemistry. 13:3774-3780.

31. Bale, M. D., L. G. Westrick, and D. F. Mosher. 1985. Incorporation of thrombospondin into fibrin clots. J. Biol. Chem. 260:75027508.

32. Sia, D. Y., D. B. Rylatt, and C. R. Parish. 1982. Anti-self receptors. V. Properties of a mouse serum factor that blocks autorosetting receptors on lymphocytes. Immunology. 45:207-216.

33. Colvin, R. B., and H. F. Dvorak. 1975. Fibrinogen/fibrin on the surface of macrophages: detection, distribution, and possible role in macrophage adherence phenomena. J. Exp. Med. 142:1377-1390.

34. Sherman, L. A., and J. Lee. 1977. Specific binding of soluble fibrin to macrophages. J. Exp. Med. 145:76-85.

35. Gonda, S. R., and J. R. Shainoff. 1982. Adsorptive endocytosis of fibrin monomer by macrophages: evidence of a receptor for the amino terminus of the fibrin $\alpha$ chain. Proc. Natl. Acad. Sci. USA. 79:45654569. 